\title{
Correction to: Transactions on Petri Nets and Other Models of Concurrency XV
}

Maciej Koutny, Fabrice Kordon (D), and Lucia Pomello

\section{Correction to:}

M. Koutny et al. (Eds.): Transactions on Petri Nets and Other Models of Concurrency XV, LNCS 12530, https://doi.org/10.1007/978-3-662-63079-2

The original version of this publication was revised. The affiliation of Lucia Pomello was corrected to "Università degli Studi di Milano-Bicocca, Milan, Italy". 